\title{
See it, try it, throw the shade guide away!
}

In the last issue of the $B D J$ you received a free sample of Tokuyama's unique Omnichroma shadeless composite. Hopefully you've already tried it and seen for yourself how it miraculously disappears when light-cured to blend seamlessly into the surrounding tooth, whatever its shade. This is because Tokuyama's unique shadelesss Omnichroma composite has innovative 'structural light' properties that means you can throw the shade guide away because the same syringe or PLT matches every tooth shade.

\section{Spherical fillers and 'structural light'}

When white light, containing all the visible colours of the spectrum, falls upon most objects, some of the colours are absorbed and only the actual colour of the object is reflected back.

Conventional composites behave just like most objects. They are only made up of irregular-shaped pigmented filler particles which give them their surface colour. Light hits the surface and reflects back with that colour. Their colour never changes.

Some objects - soap bubbles, oil on water, peacock feathers, certain breeds of butterfly etc - behave differently. They appear to have different colours depending upon how they are looked at. This is the natural phenomenon of 'structural light'.

Tokuyama's unique shadeless Omnichroma composite contains unpigmented spherical filler particles grown in a sol-gel process to a very consistent and precise $260 \mathrm{~nm}$ diameter and a clear resin. This precise spherical filler particle size provides Omnichroma with unique 'structural light' properties in the

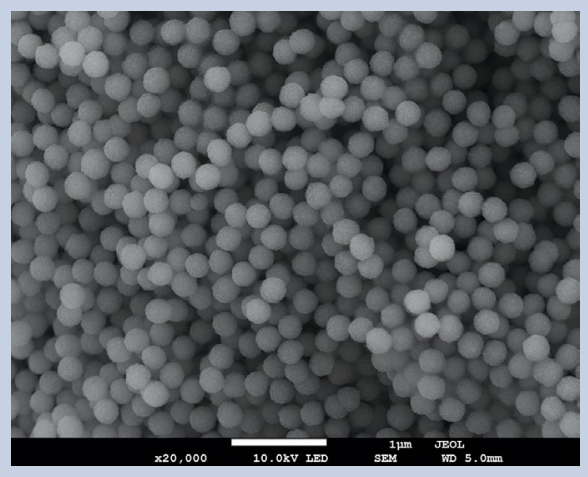

2000x SEM image of Omnichroma's $260 \mathrm{~nm}$ spherical filler particles same red/yellow range as natural teeth.

Light hitting an Omnichroma restoration passes through the clear resin and bounces back from the cavity walls with the colour of the tooth. Simultaneously, the red/ yellow 'structural light' created by the unpigmented spherical filler particles reflects back and combines with the light from the surrounding tooth to perfectly match its colour, whatever its shade!

And it never stops! If the tooth changes colour or is bleached, Omnichroma restorations automatically adapt to the colour change, unlike conventional composite materials which stand out or need replacing.

This patented technology also helps with inventory control because you only need one Omnichroma syringe or PLT, not the myriad of shades required by other brands.

It doesn't end there. Conventional composites contain filler that is both random in shape and size (gravel like) and relatively abrasive. These irregular filler particles produce an uneven surface that is difficult to polish. In addition, they frequently break away, diminishing the quality and appearance of your work.

All Tokuyama composites contain fillers that are smooth, spherical and much finer than conventional fillers, making all your Tokuyama restorations much silkier with unique optical shading properties. Not only does your restoration look better and shinier, but it lasts and lasts!

In addition, all Tokuyama composites have outstanding working, modelling and cavity adaptation characteristics, all aided by their spherical particles. They are quicker and easier to pack into nooks and crannies, reducing the risk of voids; easier to sculpt and carve, producing enhanced morphology of the final restoration; and have a much smoother surface finish with a natural high sheen that requires minimal if any polishing.

\section{Omnichroma Flow}

For clinicians who prefer flowable materials for certain indications - anterior and posterior restorations, cavity bases or lining and composite/ceramic repairs - Tokuyama created low-viscosity Omnichroma Flow.

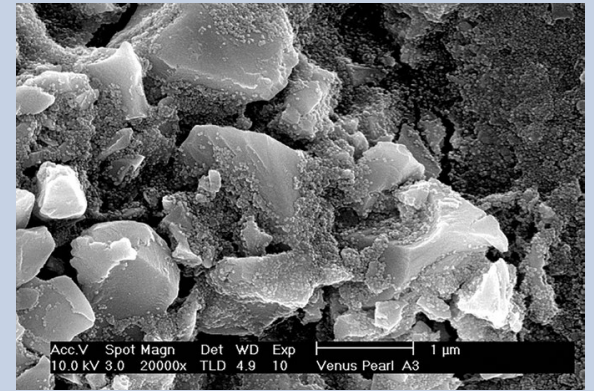

$2000 x$ SEM image of conventional irregular-shaped composite filler particles

Omnichroma Flow offers all the benefits of perfect colour matching whatever the shade, simplified inventory management, excellent cavity adaptation, non-stickiness, excellent polishability, remarkable glossiness and freedom from BisGMA and bisphenol A-related monomers.

Other award-winning Tokuyama products Whilst Omnichroma and Omnichroma Flow may not be the answer for absolutely every restoration, they will be the answer for nearly all of them. For the others, there's award-winning Tokuyama Estelite Sigma Quick (Dental Advisor's Top Universal Composite for 12 years running), Asteria layering composite for anterior restorations and Estelite Bulk Fill Flow for posterior restorations. All of which contain patented spherical filler particles of different sizes and offer outstanding shade-matching ability.

Tokuyama also offer award-winning 8th generation Universal Bond, named one of only two Dental Advisor Innovative Products of the Year 2020: the other Omnichroma.

Tokuyama Universal Bond does not require any light-curing step, saving time and reducing the risk of failed restorations due to inadequate curing. Taking just 20 seconds, it is compatible with light-curing, dual-curing and self-curing composites without any additional additives; it is also suitable for use as a primer for silica-based, zirconia-based and metallic restorations.

For more information about the complete Tokuyama range contact your local Trycare Representative, call 01274885544 or visit www.trycare.co.uk. 